\title{
Introduction to Non-Commutative Geometry
}

\author{
J. Madore \\ LPT, Batiment 211, Université de Paris-Sud \\ F-91405 Orsay (France) \\ E-mail: 'madore@th.u-psud.fri
}

ABSTRACT: A review is made of some recent results in noncommutative geometry, especially those aspects which might in some way be of use in the study of strings and membranes. Efforts to add a gravitational field to noncommutative models of space-time are also reviewed. Special emphasis is placed on the case which could be considered as the noncommutative analog of a parallelizable space-time. It is argued that, at least in this case, there is a rigid relation between the noncommutative structure of the space-time on the one hand and the nature of the gravitational field which remains as a 'shadow' in the commutative limit on the other.

\section{KEYwords: 'Noncommutative geometry, matrix calculus, gravity'}

\section{Historical introduction}

To control the divergences which from the very beginning had plagued quantum electrodynamics, Heisenberg already in the 1930's proposed to replace the space-time continuum by a lattice structure. A lattice however breaks Lorentz invariance and can hardly be considered as fundamental. It was Snyder [122i, 123i] who first had the idea of using a noncommutative structure at small length scales to introduce an effective cutoff in field theory similar to a lattice but at the same time maintaining Lorentz invariance. His suggestion came however just at the time when the renormalization program finally successfully became an effective if rather ad hoc prescription for predicting numbers from the theory of quantum electrodynamics and it was for the most part ignored. Some time later von Neumann introduced the term 'noncommutative geometry' to refer in general to a geometry in which an algebra of functions is replaced by a noncommutative algebra. As in the quantization of classical phase-space, coordinates are replaced by generators of the algebra [427]. Since these do not commute they cannot be simultaneously diagonalized and the space disappears. One can argue [90] that, just as Bohr cells replace classicalphase-space points, the appropriate intuitive no- tion to replace a 'point' is a Planck cell of dimension given by the Planck area. A noncommutative space-time looks like a solid which has a homogeneous distribution of dislocations but no disclinations. We can pursue this solid-state analogy and think of the ordinary Minkowski coordinates as macroscopic order parameters obtained by coarse-graining over scales less than the fundamental scale. They break down and must be replaced by elements of some noncommutative algebra when one considers phenomena on these scales. If a coherent description could be found for the structure of space-time which were pointless on small length scales, then the ultraviolet divergences of quantum field theory could be eliminated. In fact the elimination of these divergences is equivalent to coarse-graining the structure of space-time over small length scales; if an ultraviolet cut-off $\Lambda$ is used then the theory does not see length scales smaller than $\Lambda^{-1}$. When a physicist calculates a Feynman diagram he is forced to place a cut-off $\Lambda$ on the momentum variables in the integrands. This means that he renounces any interest in regions of space-time of volume less than $\Lambda^{-4}$. As $\Lambda$ becomes larger and larger the forbidden region becomes smaller and smaller but it can never be made to vanish. There is a fundamental length scale, much 
larger than the Planck length, below which the notion of a point is of no practical importance. The simplest and most elegant, if certainly not the only, way of introducing such a scale in a Lorentz-invariant way is through the introduction of noncommuting space-time 'coordinates'.

It might be argued that since we have made space-time 'noncommutative' we ought to do the same with the Poincaré group. This logic leads naturally to the notion of a $q$-deformed Poincaré (or Lorentz) group which acts on a very particular noncommutative version of Minkowski space called $q$-Minkowski space. The idea of a $q$-deformation goes back to the dawn of time. Almost immediately after Clifford introduced his algebras they were $q$-deformed, with $q$ a root of unity, by Sylvester [i 121,$]$. This idea was taken up later by Weyl [1299] and Schwinger [11 $\left.1 \overline{9}_{1}^{\prime}\right]$ to produce a finite version of quantum mechanics. It has also been argued, for conceptual as well as practical, numerical reasons, that the lattice version of space-time or of space is quite satisfactory if one uses a random lattice structure or graph. From this point of view the Lorentz group is a classical invariance group and is not valid at the microscopic level. A survey of this from the point of view of noncommutative geometry is to be found in the book by Landi [ $\left.8 \overline{1}^{\prime}\right]$.

Interest in Snyder's idea was revived much later when mathematicians, notably Connes [2 $2 \overline{5}$ ]

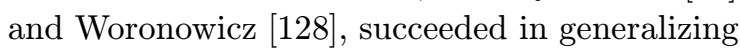
the notion of differential structure to noncommutative geometry. Just as it is possible to give many differential structures to a given topological space it is possible to define many differential calculi over a given algebra. We shall use the term 'noncommutative geometry' to mean 'noncommutative differential geometry' in the sense of Connes. Along with the introduction of a generalized integral [32] this permits one in principle to define the action of a Yang-Mills field on a large class of noncommutative geometries.

One of the more obvious applications was to the study of a modified form of Kaluza-Klein theory in which the hidden dimensions were replaced by noncommutative structures $\left[8 \overline{6}^{1}, 18 \overline{7}_{1}, \overline{4}_{4}^{4}\right]$. In simple models gravity could also be defined $\left[8 \overline{7}_{1}\right.$, 18'] although it was not until much later [105, 72.] that the technical problems involved in the definition of this field were to be to a certain extent overcome. Soon even a formulation of the standard model of the electroweak forces could be given [30]. A simultaneous development was a revival $[99,134,1,8]$ in the idea of Snyder that geometry at the Planck scale would not necessarily be described by a differential manifold.

One of the advantages of noncommutative geometry is that smooth, finite examples can be constructed which are invariant under the action of a continuous symmetry group. Such models necessarily have a minimal length associated to them and quantum field theory on them

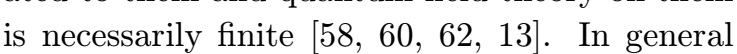
this minimal length is usually considered to be in some way or another associated with the gravitational field. The possibility which we shall consider here is that the mechanism by which this works is through the introduction of noncommuting 'coordinates'. This idea has been developed

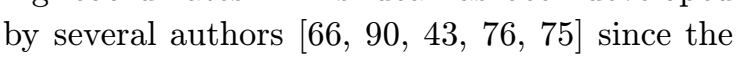
original work of Snyder. It is the left-hand arrow of the diagram

$$
\begin{array}{cc}
\mathcal{A}_{\hbar} & \Longleftarrow \Omega^{*}\left(\mathcal{A}_{\hbar}\right) \\
\Downarrow & \Uparrow \\
\text { Cut-off } & \text { Gravity }
\end{array}
$$

The $\mathcal{A}_{\hbar}$ is a noncommutative algebra and the index $k$ indicates the area scale below which the noncommutativity is relevant; this would normally be taken to be the Planck area. The top arrow is a mathematical triviality; the $\Omega^{*}\left(\mathcal{A}_{k}\right)$ is a second algebra which contains $\mathcal{A}_{k}$ and is what gives a differential structure to it. We shall give examples of this below. We shall attempt, not completely successfully, to argue that each gravitational field is the unique 'shadow' in the limit $k \rightarrow 0$ of some differential structure over some noncommutative algebra. This would define the right-hand arrow of the diagram. A hand-waving argument can be given [93- which allows one to think of the noncommutative structure of spacetime as being due to quantum fluctuations of the light-cone in ordinary 4-dimensional space-time. This relies on the existence of quantum gravitational fluctuations. A purely classical argument based on the formation of black-holes has been also given [4 $43_{1}^{\prime}$. In both cases the classical grav- 
itational field is to be considered as regularizing the ultraviolet divergences through the introduction of the noncommutative structure of spacetime. This can be strengthened as the conjecture that the classical gravitational field and the noncommutative nature of space-time are two aspects of the same thing. If the gravitational field is quantized then presumably the light-cone will fluctuate and any two points with a space-like separation would have a time-like separation on a time scale of the order of the Planck time, in which case the corresponding operators would no longer commute. So even in flat space-time quantum fluctuations of the gravitational field could be expected to introduce a non-locality in the theory. This is one possible source of noncommutative geometry on the order of the Planck scale. The composition of the three arrows in (1.1. i) is an expression of an old idea, due to Pauli, that perturbative ultraviolet divergences will somehow be regularized by the gravitational field. We refer to Garay [56 $\left.{ }^{n}\right]$ for a recent review.

One example from which one can seek inspiration in looking for examples of noncommutative geometries is quantized phase space, which had been already studied from a noncommutative point of view by Dirac [42]]. The minimal length in this case is given by the Heisenberg uncertainty relations or by modifications thereof $\left[\overline{7}_{6}\right]$. In fact in order to explain the supposed Zitterbewegung of the electron Schrödinger had proposed to mix position space with momentum space in order to obtain a set of center-of-mass coordinates which did not commute. This idea has inspired many of the recent attempts to introduce minimal lengths. We refer to [4 $4 \overline{8}, \overline{7} \overline{7}$, $]$ for examples which are in one way or another connected to noncommutative geometry. Another concept from quantum mechanics which is useful in concrete applications is that of a coherent state. This was first used in a finite noncommutative geometry by Grosse \& Prešnajder [5 $\left.5 \overline{9}_{1}^{\prime}\right]$ and later applied $\left[77_{1}, 1,1,23\right]$ to the calculation of propagators on infinite noncommutative geometries, which now become regular 2-point functions and yield finite vacuum fluctuations. Although efforts have been made in this direction [23글 these fluctuations have not been satisfactorily included as a source of the gravitational field, even in some 'quasi-commutative' approximation. If this were done then the missing arrow in (11.1) could be drawn. The difficulty is partly due to the lack of tractable noncommutative versions of curved spaces.

The fundamental open problem of the noncommutative theory of gravity concerns of course the relation it might have to a future quantum theory of gravity either directly or via the theory of 'strings' and 'membranes'. But there are more immediate technical problems which have not received a satisfactory answer. We shall mention the problem of the definition of the curvature. It is not certain that the ordinary definition of curvature taken directly from differential geometry is the quantity which is most useful in the noncommutative theory. Cyclic homology groups have been proposed by Connes as the appropriate generalization to noncommutative geometry of topological invariants; the definition of other, non-topological, invariants in not clear. It is not in fact even obvious that one should attempt to define curvature invariants.

There is an interesting theory of gravity, due to Sakharov and popularized by Wheeler, called induced gravity, in which the gravitational field is a phenomenological coarse-graining of more fundamental fields. Flat Minkowski space-time is to be considered as a sort of perfect crystal and curvature as a manifestation of elastic tension, or possibly of defects, in this structure. A deformation in the crystal produces a variation in the vacuum energy which we perceive as gravitational energy. 'Gravitation is to particle physics as elasticity is to chemical physics: merely a statistical measure of residual energies.' The description of the gravitational field which we are attempting to formulate using noncommutative geometry is not far from this. We have noticed that the use of noncommuting coordinates is a convenient way of making a discrete structure like a lattice invariant under the action of a continuous group. In this sense what we would like to propose is a Lorentz-invariant version of Sakharov's crystal. Each coordinate can be separately measured and found to have a distribution of eigenvalues similar to the distribution of atoms in a crystal. The gravitational field is to be considered as a measure of the variation of this distribution just as 
elastic energy is a measure of the variation in the density of atoms in a crystal.

When referring to the version of space-time which we describe here we use the adjective 'fuzzy' to underline the fact that points are ill-defined. Since the algebraic structure is described by commutation relations the qualifier 'quantum'

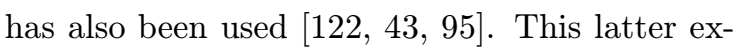
pression is unfortunate since the structure has no immediate relation to quantum mechanics and also it leads to confusion with 'spaces' on which 'quantum groups' act. To add to the confusion the word 'quantum' has also been used [5기] to designate equivalence classes of ordinary differential geometries which yield isomorphic string theories and the word 'lattice' has been used li22', i4', '124'] to designate what we here qualify as 'fuzzy'.

\section{The basic idea}

The basic idea is to 'quantize' the coordinates $x^{\mu}$ of Minkowski space-time, to replace them by generators $q^{\mu}$ of a noncommutative algebra $\mathcal{A}_{\hbar}$ :

$$
\left[q^{\mu}, q^{\nu}\right]=i \hbar q^{\mu \nu}, \quad \hbar \simeq \mu_{P}^{-2}=G \hbar .
$$

We have written the area scale as an index but of course this in no way characterizes the algebra. The latter would be restricted but not in general uniquely defined by the structure of $q^{\mu \nu}$. The equivalent of the Heisenberg uncertainty relations would be $\Lambda^{2} \hbar \lesssim 1$. Very roughly speaking then a fuzzy space-time would be composed of cells of volume $V \simeq(2 \pi k)^{2}$. In the limit $\mu_{P} \rightarrow \infty$ we shall suppose that $q^{\mu} \rightarrow x^{\mu}$ but one could expect perhaps a singular 'renormalization constant' $Z: q^{\mu} \rightarrow Z x^{\mu}$. To define the theory it would be important to introduce a state and to identify the $q^{\mu}$ as hermitian operators on some Hilbert space.

As a simple example it is interesting to consider the phase space of a particle in a plane: $\left(q^{1}, q^{2}, p_{1}, p_{2}\right)$. In classical mechanics one has four commuting operators; in quantum mechanics one has the commutation relations

$$
\left[q^{1}, p_{1}\right]=i \hbar, \quad\left[q^{2}, p_{2}\right]=i \hbar .
$$

The points of classical phase space have been replaced by 'Bohr cells' of area $2 \pi \hbar$. Consider the divergent integral

$$
I=\int \frac{d p_{1} d p_{2}}{p^{2}}, \quad p^{2}=p_{1}^{2}+p_{2}^{2} .
$$

If one introduces a magnetic field $B$ normal to the plane then the appropriately modified gaugecovariant momenta no longer commute:

$$
\left[p_{1}, p_{2}\right]=i \hbar e B
$$

The points of momentum space have been replaced by 'Landau cells' of area $\hbar e B$. This serves in general as an infrared cut-off:

$$
p^{2} \gtrsim \hbar e B
$$

If one were to replace the magnetic field by a gaussian curvature $K$, $\hbar e B \mapsto \hbar^{2} K$ then one would have the same effect; curvature in general acts as a mass.

In this example 'quantizing' position-space coordinates consists in replacing them by two operators which satisfy a commutation relation of the form

$$
\left[q^{1}, q^{2}\right]=i \hbar q^{12}
$$

Ipso facto the points of position space are replaced by 'Planck cells' of area $2 \pi \hbar$ and the integral $I$ is completely regularized:

$$
I \sim \log (\hbar K) .
$$

This vague idea can actually be implemented by

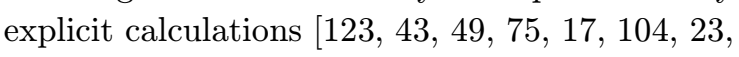
i78. There is now however a new complication. Although $\hbar$ is a constant and one usually supposes the magnetic field $B$ to be independent of the momenta, the operator $q^{12}$ in general could be expected a priori to be an arbitrary element of the algebra $\mathcal{A}_{k}$. Our working assumption is that there is a one-to-one correspondence between the $q^{12}$ and the classical 'gravitational' field of the commutative limit. In Wheeler's language the $q^{12}$ determines the 'lattice' spacings away from (flat space) equilibrium. For example, with the definition of metric which we shall advocate one finds that if $q^{12}=1$ the surface is flat, if $r q^{12}=$ $i q^{3}$ with $\sum\left(q^{i}\right)^{2}=r^{2}$ the surface is a sphere and if $k q^{12}=h\left(q^{2}\right)^{2}$ the surface is a pseudosphere. If $q^{12}$ does not belong to the center of the algebra then Jacobi identities will imply that the canonical commutation relations $(2.1)$ will be modified. 
As a simple illustration of how a 'space' can be 'discrete' in some sense and still covariant under the action of a continuous symmetry group one can consider the ordinary round 2-sphere, which has acting on it the rotational group $\mathrm{SO}_{3}$. As a simple example of a lattice structure one can consider two points on the sphere, for example the north and south poles. One immediately notices of course that by choosing the two points one has broken the rotational invariance. It can be restored at the expense of commutativity. The set of functions on the two points can be identified with the algebra $\mathbb{C} \times \mathbb{C}$ of diagonal $2 \times 2$ matrices, each of the two entries on the diagonal corresponding to a possible value of a function at one of the two points. Now an action of a group on the lattice is equivalent to an action of the group on the matrices and there can obviously be no (non-trivial) action of the group $\mathrm{SO}_{3}$ on $\mathbb{C} \times \mathbb{C}$. However, if one extends the algebra to the noncommutative algebra $M_{2}(\mathbb{C})$ of all $2 \times 2$ matrices one recovers the invariance. The two points, so to speak, have been smeared out over the surface of a sphere; they are replaced by two cells. An 'observable' is an hermitian $2 \times 2$ matrix and has therefore two real eigenvalues, which are its values on the two cells. Although what we have just done has nothing to do with Planck's constant it is similar to the procedure of replacing a classical spin which can take two values by a quantum spin of total spin $1 / 2$. Only the quantum spin is invariant under the rotation group.

By replacing the spin $1 / 2$ by arbitrary spin $s$ one can describe a 'lattice structure' of $n=2 s+1$ points in an $\mathrm{SO}_{3}$-invariant manner. The algebra becomes then the algebra $M_{n}$ of $n \times n$ complex matrices and there are $n$ cells of area $2 \pi \hbar$ with

$$
n \simeq \frac{\operatorname{Vol}\left(S^{2}\right)}{2 \pi \hbar} .
$$

In general, a static, closed surface in a fuzzy space-time as we define it can only have a finite number of modes and will be described by some finite-dimensional algebra $\left[\overline{5} \overline{8}_{v}^{\prime}, \overline{6} \overline{0}_{n}^{\prime}, \overline{6} \overline{2}_{v}^{\prime}, \overline{6} \overline{3}_{n}^{\prime}\right.$ i64i]. Graded extensions of some of these algebras have also been constructed $[6 \overline{5}, 6 \overline{1}, 1]$. Although we are interested in a matrix version of surfaces primarily as a model of an eventual noncommutative theory of gravity they have a certain inter- est in other, closely related, domain of physics. We have seen, for example, that without the differential calculus, to be introduced below, the fuzzy sphere is basically just an approximation to a classical spin $r$ by a quantum spin $r$ with $\hbar$ in lieu of $\hbar$. It has been extended in various directions under various names and for various

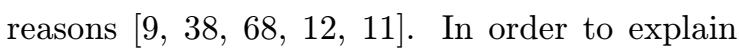
the finite entropy of a black hole it has been conjectured, for example by 't Hooft [1 $12 \overline{2}$, that the horizon has a structure of a fuzzy 2 -sphere since the latter has a finite number of 'points' and yet has an $\mathrm{SO}_{3}$-invariant geometry. The horizon of a black hole might be a unique situation in which one can actually 'see' the cellular structure of space.

\section{Differential calculi}

In the following sections we shall discuss the algebra $\Omega^{*}(\mathcal{A})$ of $(1,1)$ and the associated differential $d$, which satisfies the relation $d^{2}=0$. The couple $\left(\Omega^{*}(\mathcal{A}), d\right)$ is known as a differential calculus over the algebra $\mathcal{A}$. The algebra $\mathcal{A}$ is what in ordinary geometry would determine the set of points one is considering, with possibly an additional topological or measure theoretic structure. The differential calculus is what gives an additional differential structure or a notion of smoothness. On a commutative algebra of functions on a lattice, for example, it would determine the number of nearest neighbours and therefore the dimension. We give some simple examples before stating the general definition.

\subsection{The Connes-Lott model}

Write $\mathbb{C}^{3}=\mathbb{C}^{2} \oplus \mathbb{C}^{1}$ and decompose $M_{3}=M_{3}(\mathbb{C})$ accordingly:

$$
M_{3}=M_{3}^{+} \oplus M_{3}^{-}, \quad M_{3}^{+}=M_{2} \times \mathbb{C} .
$$

Fix

$$
\eta=\left(\begin{array}{ccc}
0 & 0 & a_{1} \\
0 & 0 & a_{2} \\
-a_{1}^{*} & -a_{2}^{*} & 0
\end{array}\right), \quad \eta\left(=-\eta^{*}\right) \in M_{3}^{-} .
$$

For arbitrary $\alpha \in M_{3}$ define $d \alpha=-[\eta, \alpha]$ with a graded bracket. Then $d$ is a graded derivation of $M_{3}$. In particular one finds that

$$
d \eta=-2 \eta^{2}, \quad d^{2} \alpha=\left[\eta^{2}, \alpha\right] .
$$


We define an algebra

$$
\Omega_{\eta}^{*}=\Omega_{\eta}^{0} \oplus \Omega_{\eta}^{1} \oplus \Omega_{\eta}^{2}
$$

where

$$
\Omega_{\eta}^{0}=M_{3}^{+}, \quad \Omega_{\eta}^{1}=M_{3}^{-}, \quad \Omega_{\eta}^{2}=\mathbb{C}
$$

and by $\mathbb{C}$ we mean the second factor of $M_{3}^{+}$, the entry in the lower right-hand corner of $M_{3}$. This definition of $\Omega_{\eta}^{2}$ is the largest one which is consistent with the condition that $d^{2}=0$ since $\left[\eta^{2}, \alpha\right]$ necessarily belongs to the first factor of $M_{3}^{+}$if $\alpha \in \Omega_{\eta}^{0}$. The multiplication law which defines the algebra is ordinary matrix multiplication. It is possible to chose $\eta$ such that

$$
d \eta+\eta^{2}=1
$$

The right-hand side of this equation is to be considered as an element of $\Omega_{\eta}^{2}$. The algebra can be extended to a $\mathbb{Z}$-graded algebra by setting $\Omega_{\eta}^{p}=0$ for $p \geq 3$.

\subsection{A matrix model}

Consider $M_{n}$ and over it the algebra

$$
\Omega_{u}^{*}\left(M_{n}\right)=\bigoplus_{i=1}^{\infty} \Omega_{u}^{i}\left(M_{n}\right)
$$

where $\Omega_{u}^{i}\left(M_{n}\right)$ is a submodule of the tensor product of $i+1$ copies of $M_{n}$ and the product is ordinary matrix multiplication of adjoining factors. Define a map $d_{u}$ of $\Omega^{0}\left(M_{n}\right)=M_{n}$ into $M_{n} \otimes M_{n}$ by

$$
d_{u} f=1 \otimes f-f \otimes 1, \quad f \in M_{n} .
$$

Then $\Omega^{1}\left(M_{n}\right)$ is by definition the $M_{n}$-bimodule generated by the image of $d_{u}$. We use the symbol $f$ to underline the role of the elements of $M_{n}$ as 'functions'. There is a natural extension of $d_{u}$ to all of $\Omega^{*}\left(M_{n}\right)$ such that $d_{u}^{2}=0$. The couple $\left(\Omega_{u}^{*}\left(M_{n}\right), d_{u}\right)$ is known as the universal calculus over $M_{n}$. It can be constructed over an arbitrary associative algebra with unit.

Introduce an antihermitian basis $\lambda_{a}$ of $S U_{n}$ and define

$$
\theta_{u}^{a}=\lambda_{b} \lambda^{a} d_{u} \lambda^{b}
$$

Then one can show that

$$
\theta_{u}^{a} f=f \theta_{u}^{a}, \quad f \in M_{n}
$$

Define an algebra $\Omega^{*}\left(M_{n}\right)$ by imposing the relations

$$
\theta^{a} \theta^{b}=-\theta^{b} \theta^{a}, \quad \theta^{a}=\theta_{u}^{a}
$$

and a differential $d$ as the restriction of $d_{u}$. It is easily seen that

$$
\Omega^{1}\left(M_{n}\right) \simeq \bigoplus_{i=1}^{n^{2}-1} M_{n}
$$

and one can show that

$$
d \theta^{a}=-\frac{1}{2} C^{a}{ }_{b c} \theta^{b} \theta^{c} .
$$

Introduce $\theta=-\lambda_{a} \theta^{a}$. Then one sees that

$$
d f=-[\theta, f]
$$

and that

$$
d \theta+\theta^{2}=0 .
$$

There is an obvious similarity between $\Omega^{*}\left(M_{n}\right)$ and the algebra of de Rham differential forms on the group $S U_{n}$.

\subsection{The noncommutative 2-torus}

The algebra $\mathcal{P}(u, v)$ of polynomials in $u=e^{i x}$, $v=e^{i y}$ is dense in any algebra of functions on the torus, defined by the relations $0 \leq x \leq 2 \pi$, $0 \leq y \leq 2 \pi$, where $x$ and $y$ are the ordinary cartesian coordinates of $\mathbb{R}^{2}$. If one considers a square lattice of $n^{2}$ points then $u^{n}=1$ and $v^{n}=$ 1 and the algebra is reduced to a subalgebra $\mathcal{P}_{n}$ of dimension $n^{2}$. Introduce a basis $|j\rangle_{1}, 0 \leq j \leq$ $n-1$, of $\mathbb{C}^{n}$ with $|n\rangle_{1} \equiv|0\rangle_{1}$ and replace $u$ and $v$ by the operators

$$
u|j\rangle_{1}=q^{j}|j\rangle_{1}, \quad v|j\rangle_{1}=|j+1\rangle_{1}, \quad q^{n}=1 .
$$

Then the new elements $u$ and $v$ satisfy the relations

$$
u v=q v u, \quad u^{n}=1, \quad v^{n}=1
$$

and the algebra they generate is the matrix algebra $M_{n}$ instead of the commutative algebra $\mathcal{P}_{n}$. There is also basis $|j\rangle_{2}$ in which $v$ is diagonal and

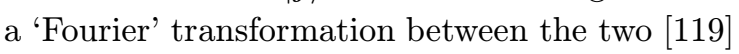
Introduce the forms [i $[\bar{i}]$

$$
\begin{aligned}
& \theta^{1}=-i\left(1-\frac{n}{n-1}|0\rangle_{2}\langle 0|\right) u^{-1} d u, \\
& \theta^{2}=-i\left(1-\frac{n}{n-1}|n-1\rangle_{1}\langle n-1|\right) v^{-1} d v .
\end{aligned}
$$


One can verify the relations

$$
\theta^{a} f=f \theta^{a}, \quad \theta^{a} \theta^{b}=-\theta^{b} \theta^{a} .
$$

It follows that

$$
\Omega^{1}\left(M_{n}\right) \simeq \bigoplus_{1}^{2} M_{n}, \quad d \theta^{a}=0 .
$$

The differential calculus has the form one would expect of a noncommutative version of the torus.

\subsection{The quantum line $\mathbb{R}_{q}^{1}$}

The quantum line $\left[\underline{4} \overline{7}_{1}, 1\right]$ mal algebra with involution with two generators $x$ and $\Lambda$ which satisfy the relations

$$
x=x^{*}, \quad \Lambda \Lambda^{*}=1, \quad x \Lambda=q \Lambda x
$$

for some real number $q$. There are two natural differential calculi $\left(\Omega^{*}\left(\mathbb{R}_{q}^{1}\right), d\right)$ and $\left(\bar{\Omega}^{*}\left(\mathbb{R}_{q}^{1}\right), \bar{d}\right)$ which are defined by the relations

$$
\begin{array}{ll}
d \Lambda=0, & \\
d x \Lambda=q \Lambda d x, & x d x=q d x x, \\
\bar{d} x \Lambda=q \Lambda \bar{d} x, \quad x \bar{d} x=q^{-1} \bar{d} x x .
\end{array}
$$

If one introduces the element $\theta^{1} \in \Omega^{1}\left(\mathbb{R}_{q}^{1}\right)$ and $\bar{\theta}^{1} \in \bar{\Omega}^{1}\left(\mathbb{R}_{q}^{1}\right)$ defined by

$$
\theta^{1}=\Lambda^{-1} x^{-1} d x, \quad \bar{\theta}^{1}=q^{-1} \Lambda x^{-1} \bar{d} x
$$

then one finds that

$$
\theta^{1} f=f \theta^{1}, \quad \bar{\theta}^{1} f=f \bar{\theta}^{1}, \quad f \in \mathbb{R}_{q}^{1} .
$$

It follows that

$$
\Omega^{1}\left(\mathbb{R}_{q}^{1}\right) \simeq \mathbb{R}_{q}^{1}, \quad \bar{\Omega}^{1}\left(\mathbb{R}_{q}^{1}\right) \simeq \mathbb{R}_{q}^{1} .
$$

There is a simple representation of $\mathbb{R}_{q}^{1}$ on an infinite-dimensional analogue of the basis which we introduced to represent the noncommutative torus. It is given by

$$
x|k\rangle=q^{k}|k\rangle, \quad \Lambda|k\rangle=|k+1\rangle
$$

A partial classification exists [1] 1 15 - of all representations. The representation can be extended to a representation of the differential calculi by the identifications [1는

$$
\theta^{1} \mapsto 1, \quad \bar{\theta}^{1} \mapsto 1 .
$$

There are many other infinite-dimensional algebras with associated differential calculi which have served as basis for exploring the possible applications of noncommutative geometry to physics. We mention in particular the noncommuta-

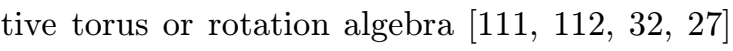
which extends the noncommutative torus we defined above, the quantum plane [10 $1,102,1 \overline{1} \overline{1} \overline{1}]$,

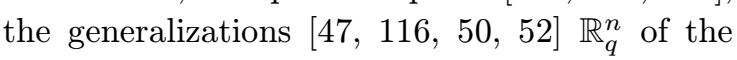
quantum line, the quantum sphere [107] the jor-

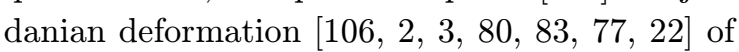
$S L(2, \mathbb{C})$ as well as 'quantum' deformations of

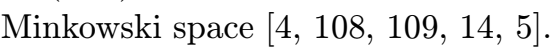

\subsection{Differential calculi in general}

Consider an associative algebra $\mathcal{A}$ with a unit and a graded algebra

$$
\Omega^{*}(\mathcal{A})=\bigoplus_{i \geq 0} \Omega^{i}(\mathcal{A}), \quad \Omega^{0}(\mathcal{A})=\mathcal{A}
$$

which is the direct sum of a family of $\mathcal{A}$-bimodules. A differential $d$ is a graded derivation of $\Omega^{*}(\mathcal{A})$ with $d^{2}=0$. If $\alpha \in \Omega^{i}(\mathcal{A})$ and $\beta \in \Omega^{j}(\mathcal{A})$ then $\alpha \beta \in \Omega^{i+j}(\mathcal{A})$ and $d(\alpha \beta) \in \Omega^{i+j+1}(\mathcal{A})$ with

$$
d(\alpha \beta)=d \alpha \beta+(-1)^{i} \alpha d \beta .
$$

One usually supposes that as a bimodule $\Omega^{1}(\mathcal{A})$ is generated by the image of $d$. A differential algebra is a graded algebra with a differential. We have already noted that the couple $\left(\Omega^{*}(\mathcal{A}), d\right)$ is called a differential calculus over $\mathcal{A}$. An element of $\Omega^{p}(\mathcal{A})$ is known as a $p$-form.

Let $\Omega^{1}(\mathcal{A})$ be an arbitrary $\mathcal{A}$-bimodule and let $d$ be a module morphism

$$
\mathcal{A} \stackrel{d}{\longrightarrow} \Omega^{1}(\mathcal{A})
$$

from $\mathcal{A}$ into $\Omega^{1}(\mathcal{A})$. We have mentioned already the universal calculus $\Omega_{u}^{*}(\mathcal{A})$ over $\mathcal{A}$. In particular it defines a map

$$
\mathcal{A} \stackrel{d_{u}}{\longrightarrow} \mathcal{A} \otimes \mathcal{A}
$$

by

$$
d_{u} f=1 \otimes f-f \otimes 1, \quad f \in \mathcal{A} .
$$

As in the matrix case, by definition $\Omega_{u}^{1}(\mathcal{A}) \subset$ $\mathcal{A} \otimes \mathcal{A}$ is the smallest $\mathcal{A}$-bimodule which contains the image of $d_{u}$. We can define then a map

$$
\Omega_{u}^{1}(\mathcal{A}) \stackrel{\phi_{1}}{\longrightarrow} \Omega^{1}(\mathcal{A})
$$


of $\Omega_{u}^{1}(\mathcal{A})$ onto $\Omega^{1}(\mathcal{A})$ by

$$
\phi_{1}\left(d_{u} f\right)=d f .
$$

Because $d 1=0$ the map is well defined. We have

$$
\begin{array}{cc}
\mathcal{A} \stackrel{d_{u}}{\longrightarrow} \Omega_{u}^{1}(\mathcal{A}) \\
\| \quad \phi_{1} \downarrow \\
\mathcal{A} \stackrel{d}{\longrightarrow} \Omega^{1}(\mathcal{A})
\end{array}
$$

and we can identify

$$
\Omega^{1}(\mathcal{A})=\Omega_{u}^{1}(\mathcal{A}) / \operatorname{Ker} \phi_{1} .
$$

Every bimodule of 1-forms can be so written.

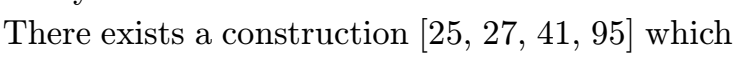
defines a differential calculus as the largest differential algebra consistent with the module structure of the 1-forms. In particular the map $\phi_{1}$ can be extended to a map

$$
\Omega_{u}^{*}(\mathcal{A}) \stackrel{\phi_{*}}{\longrightarrow} \Omega^{*}(\mathcal{A})
$$

uniquely defined by the bimodule $\Omega^{1}(\mathcal{A})$. We shall use mainly 1 -forms but on occasion 2 -forms and we shall need to introduce the product:

$$
\Omega^{1}(\mathcal{A}) \otimes_{\mathcal{A}} \Omega^{1}(\mathcal{A}) \stackrel{\pi}{\longrightarrow} \Omega^{2}(\mathcal{A}) .
$$

\subsection{Differential calculi over algebras of func- tions}

As an example let $\mathcal{A}=\mathcal{C}(V)$ be the algebra of smooth functions over a smooth manifold $V$. One can introduce the de Rham differential calculus $\Omega^{1}(\mathcal{A}) \equiv \Omega^{1}(V)$. If $f \in \mathcal{A}$ then $d_{u} f$ is the function of 2 variables

$$
d_{u} f(x, y)=f(y)-f(x) .
$$

As we have already noticed it can be defined over any algebra of functions. The de Rham 1-form $d f$ can be written locally as $d f=\partial_{\lambda} f d x^{\lambda}$ in terms of the differential of the coordinates. One can expand the function $f(y)$ about the point $x$ :

$$
f(y)=f(x)+\left(x^{\lambda}(y)-x^{\lambda}(x)\right) \partial_{\lambda} f+\cdots
$$

The map $\phi_{1}$ is given then by

$$
\phi_{1}\left(x^{\lambda}(y)-x^{\lambda}(x)\right)=d x^{\lambda} .
$$

It annihilates any $f(x, y) \in \Omega_{u}^{1}(\mathcal{A})$ of second order in $x-y$. One such form is $f d_{u} g-d_{u} g f$. In fact

$$
\begin{aligned}
& \left(f d_{u} g-d_{u} g f\right)(x, y)= \\
& \quad-(f(y)-f(x))(g(y)-g(x)) .
\end{aligned}
$$

The left-hand side does not vanish in $\Omega_{u}^{1}(\mathcal{A})$ but its image in $\Omega^{1}(\mathcal{A})$ under $\phi_{1}$ is equal to zero. The de Rham differential calculus can be characterized in fact by the condition that $f d g-d g f=0$ for arbitrary $f, g \in \mathcal{C}(V)$. It follows immediately then that $d f d g+d g d f=0$ and one readily sees in this example how the module structure of the 1 -forms influences the algebraic structure of the algebra of forms.

\subsection{The free-module case}

In order to analyze in more detail the structure of a differential calculus over an algebra $\mathcal{A}$ we shall make three assumptions. We shall suppose first that as a bimodule

$$
\Omega^{1}(\mathcal{A}) \simeq \bigoplus_{1}^{d} \mathcal{A}
$$

This is a condition which in ordinary geometry would mean that the manifold in question is parallelizable. It states in this case that globally a covariant vector can be identified with $d$ functions. An example of such a manifold is the sphere $S^{3}$. If also the manifold is topologically trivial then any covariant vector can be identified with its $d$ components. A more restrictive assumption is that there exists $d 1$-forms $\theta^{a}$ such that

$$
\left[f, \theta^{a}\right]=0, \quad 1 \leq a \leq d .
$$

We shall refer to $\theta^{a}$ as a frame or Stehbein. The existence of the frame obviously implies the parallizability assumption. As a third condition we suppose a 'smoothness' condition. We assume that there exist $d$ elements $\lambda_{a}$ of $\mathcal{A}$ such that the frame is dual to the associated derivations:

$$
\theta^{a}\left(e_{b}\right)=\delta_{b}^{a}, \quad e_{a}=\operatorname{ad} \lambda_{a} .
$$

It follows in particular that

$$
d f=e_{a} f \theta^{a}=\left[\lambda_{a}, f\right] \theta^{a}=-[\theta, f]
$$


and therefore that there exists a 'Dirac operator'

$$
\theta=-\lambda_{a} \theta^{a}
$$

Let $\theta^{\alpha}=\theta_{\lambda}^{\alpha} d x^{\lambda}$ be a moving frame on a manifold and let $\gamma^{\alpha}=\theta_{\lambda}^{\alpha} \gamma^{\lambda}$ be the associated Dirac matrices. The one can 'almost' make the identification $\theta^{\alpha} \mapsto \gamma^{\alpha}$. In fact the $\theta^{\alpha}$ anticommute and the $\gamma^{\alpha}$ almost anticommute. The designation 'Dirac operator' for $\theta$ comes from this identification. It yields the identity

$$
d f=\theta^{\alpha} e_{\alpha} f=\gamma^{\alpha} e_{\alpha} f=-[\not \not D, f]
$$

where $e_{\alpha}$ are the vector fields (derivations) dual to the moving frame. In the Connes-Lott example of Section 3.1 we noticed that it was necessary to restrict the module of 2 -forms in order to have $d^{2}=0$. This restriction is related to the problems arising from the identification of the frame with the Dirac matrices. From (13.2) it follows in fact that

$$
d^{2} f=-\left[\not D^{2}, f\right]
$$

if one uses a graded commutator.

It is not always possible to impose the 'smoothness' condition. The existence of the set of $\lambda_{a}$ imposes the consistency condition

$$
2 \lambda_{c} \lambda_{d} P_{a b}^{c d}-\lambda_{c} F_{a b}^{c}-K_{a b}=0 .
$$

This gives to the set of $\lambda_{a}$ a sort of twisted Liealgebra structure with a central extension. The coefficients lie all in the center $\mathcal{Z}(\mathcal{A})(\equiv \mathbb{C})$. The $P^{c d}{ }_{a b}$ are defined by the product in $\Omega^{*}(\mathcal{A})$ :

$$
\theta^{a} \theta^{b}=\pi\left(\theta^{a} \otimes \theta^{b}\right)=P_{c d}^{a b} \theta^{c} \otimes \theta^{d} .
$$

The $F_{a b}^{c}$ are related to the 2 -form $d \theta^{a}$ :

$$
d \theta^{a}=-\frac{1}{2} C_{b c}^{a} \theta^{b} \theta^{c}
$$

with

$$
C^{a}{ }_{b c}=F_{b c}^{a}-2 \lambda_{e} P^{(a e)} b c .
$$

The $K_{a b}$ are related to the curvature of the 'Dirac operator':

$$
d \theta+\theta^{2}=\frac{1}{2} K_{a b} \theta^{a} \theta^{b}
$$

The Connes-Lott model of Section 3.1 is not parallelizable but the matrix model of Section 3.2 and the noncommutative torus of Section 3.3 are even 'smooth'. The quantum line is 'smooth', as is $\mathbb{R}_{q}^{3}\left[{ }^{5} 2^{2}\right]$. Over the quantum plane mentioned in Section 3.4 one can construct an infinite number of 'smooth' differential calculi for each value of $d\left[4 \overline{1}_{1}\right]$. In particular, when $d=2$ and $q^{4} \neq 1$ the choice

$$
\begin{aligned}
& \lambda_{1}=\frac{1}{q^{4}-1} x^{-2} y^{2}, \\
& \lambda_{2}=\frac{1}{q^{4}-1} x^{-2}
\end{aligned}
$$

yields the Wess-Zumino calculus [1] 127 ] which has special covariance properties. The jordanian deformation is smooth [222]

If the algebra $\mathcal{A}$ is a $*$-algebra then a 'smooth' differential calculus is real if the $\lambda_{a}$ are antihermitian. The involution can be extended to the differential calculus such that

$$
d f^{*}=(d f)^{*} .
$$

The frame is hermitian and the Dirac operator is antihermitian

$$
\left(\theta^{a}\right)^{*}=\theta^{a}, \quad \theta^{*}=-\theta
$$

When

$$
P^{a b}{ }_{c d}=\frac{1}{2}\left(\delta_{c}^{a} \delta_{d}^{b}-\delta_{c}^{b} \delta_{d}^{a}\right)
$$

then the $F_{a b}^{c}$ are hermitian and the $K_{a b}$ are antihermitian.

If one has a representation [i $\left.\overline{2} \overline{7}_{1}\right]$ of the algebra and the differential calculus as von Neumann algebras then one can use the modular conjugation operator $J$ to introduce a reality condition [2] under more general conditions.

\section{Noncommutative Yang-Mills the- ory}

The group of unitary elements of the algebra of functions on a manifold is the local gauge group of electromagnetism and the covariant derivative associated to the electromagnetic potential is a map

$$
\mathcal{H} \stackrel{D}{\longrightarrow} \Omega^{1}(V) \otimes_{\mathcal{A}} \mathcal{H}
$$

from a $\mathcal{C}(V)$-module $\mathcal{H}$ to the tensor product $\Omega^{1}(V) \otimes_{\mathcal{C}(V)} \mathcal{H}$, which satisfies a Leibniz rule $D(f \psi)=d f \otimes \psi+f D \psi, \quad f \in \mathcal{C}(V), \quad \psi \in \mathcal{H}$. 
We shall often omit the tensor-product symbol in the following. As far as the electromagnetic potential is concerned we can identify $\mathcal{H}$ with $\mathcal{C}(V)$ itself; electromagnetism couples equally, for example, to all four components of a Dirac spinor. The covariant derivative is defined therefore by the Leibniz rule and the definition

$$
D 1=A \otimes 1=A
$$

In the following we shall study electromagnetism on some noncommutative geometries. We emphasize the fact that it is electromagnetism; the geometry has changed not the theory being studied. Because of the noncommutativity however the result often looks more like nonabelian Yang-Mills theory and so we refer to it as such. The essential difference lies in the fact that one must now distinguish between left- and rightmodule structures.

\subsection{The Connes-Lott model}

Choose as left module $\mathcal{H}=\mathbb{C}^{3}$ and let $\psi \in \mathcal{H}$. Consider an anti-hermitian 1-form $\omega$, element of $\Omega_{\eta}^{1}$ introduced in Section 3.1. The gauge group is the group $U_{2} \times U_{1}$ of unitary elements of $M_{3}^{+}$. We repeat that this is the gauge group of 'local' electromagnetic gauge transformations on the 'space' described by the algebra $M_{3}^{+}$. Let $g \in U_{2} \times U_{1}$ be a gauge transformation. It is easy to see that

$$
\eta^{\prime}=g^{-1} \eta g+g^{-1} d g=g^{-1} \eta g-g^{-1}[\eta, g]=\eta .
$$

This is a particular property of noncommutative geometry and is due to the fact that, at least in the geometries which we shall study, the 'Dirac operator' is itself a 1-form. In more sophisticated geometries [2] $\left.\bar{Z}_{1}\right]$ this will be in general no longer the case. If we write

$$
\omega=\eta+\phi
$$

then it is easy to see that

$$
\phi^{\prime}=g^{-1} \phi g
$$

The $\phi$ transforms under the adjoint representation of $U_{2} \times U_{1}$. If we consider $\omega$ as a connection form then its curvature or field strength is given by

$$
\Omega=d \omega+\omega^{2}=1+\phi^{2}=1-|\phi|^{2} .
$$

We recall that in this geometry a 2 -form can be considered as a complex number. The unit on thee right-hand side is the unit in the right-hand side of $(3.1)$. The action is given by

$$
V(\phi)=\frac{1}{4} \operatorname{Tr}\left(1-|\phi|^{2}\right)^{2} .
$$

This describes electromagnetism on the ConnesLott geometry.

A covariant derivative can be defined as

$$
D \psi=\phi \psi \text {. }
$$

Although this is gauge covariant it is not in the strict sense of the word a covariant derivative. We shall return again to this point below.

\subsection{The matrix model}

The same considerations can be repeated using the geometry of Section 3.2 with similar results. We choose as left module $\mathcal{H}=M_{n}$. The gauge group is now $U_{n} \subset M_{n}$. Let $g \in U_{n}$. It is easy to see that

$$
\theta^{\prime}=g^{-1} \theta g+g^{-1} d g=g^{-1} \theta g-g^{-1}[\theta, g]=\theta .
$$

If we write then an arbitrary connection form $\omega$ as $\omega=\theta+\phi$ we find that $\phi$ transforms under the adjoint representation of $U_{n}$. The curvature or field strength is given by

$$
\Omega=\frac{1}{2} \Omega_{a b} \theta^{a} \theta^{b}, \quad \Omega_{a b}=\left[\phi_{a}, \phi_{b}\right]-C_{a b}^{c} \phi_{c}
$$

and the associated action is given by

$$
V(\phi)=-\frac{1}{4} \operatorname{Tr}\left(\Omega_{a b} \Omega^{a b}\right) .
$$

This describes electromagnetism on the matrix geometry. The action vanishes at $\phi=0$ and on the gauge orbit $\phi=g^{-1} \theta g$ of $\theta$.

A covariant derivative can be defined by one of the expressions

$$
\begin{aligned}
& D \psi=\phi \psi . \\
& D \psi=\psi \phi . \\
& D \psi=[\phi, \psi] .
\end{aligned}
$$

Although these are all gauge covariant they are not in the strict sense of the word covariant derivatives. The derivative

$$
D \psi=-\theta \psi-\psi \phi
$$


is covariant from the left and gauge-covariant from the right and the derivative

$$
D \psi=\psi \theta+\phi \psi
$$

is covariant from the right and gauge-covariant from the left.

At least in a particular case $\left[\overline{9} \overline{6}_{n}^{\prime}\right]$ the model of the previous section can be obtained as a singular contraction of the model presented here. Electromagnetism has been studied also on infinitedimensional noncommutative algebras. In fact the first example [32] was on the rotation algebra.

\section{Metrics}

We shall define a metric as a bilinear map

$$
\Omega^{1}(\mathcal{A}) \otimes_{\mathcal{A}} \Omega^{1}(\mathcal{A}) \stackrel{g}{\longrightarrow} \mathcal{A} .
$$

This is a 'conservative' definition, a straightforward generalization of one of the possible definitions of a metric in ordinary differential geometry. The usual definition of a metric in the commutative case is a bilinear map

$$
\mathcal{X} \otimes_{\mathcal{C}(V)} \mathcal{X} \stackrel{g}{\longrightarrow} \mathcal{C}(V)
$$

where $\mathcal{X}$ is the $\mathcal{C}(V)$-bimodule of vector fields on $V$. This definition is not suitable in the noncommutative case since the set of derivations of the algebra, which is the generalization of $\mathcal{X}$, has no natural structure as an $\mathcal{A}$-module. The linearity condition is equivalent to a locality condition for the metric; the length of a vector at a given point depends only on the value of the metric and the vector field at that point. In the noncommutative case bilinearity is the natural (and only possible) expression of locality. It would exclude, for example, a metric in ordinary geometry defined by a map of the form

$$
g(\alpha, \beta)(x)=\int_{V} g_{x}\left(\alpha_{x}, \beta_{y}\right) G(x, y) d y .
$$

Here $\alpha, \beta \in \Omega^{1}(V)$ and $g_{x}$ is a metric on the tangent space at the point $x \in V$. The function $G(x, y)$ is an arbitrary smooth function of $x$ and $y$ and $d y$ is the measure on $V$ induced by the metric.
Introduce a bilinear flip $\sigma$ :

$$
\Omega^{1}(\mathcal{A}) \otimes_{\mathcal{A}} \Omega^{1}(\mathcal{A}) \stackrel{\sigma}{\longrightarrow} \Omega^{1}(\mathcal{A}) \otimes_{\mathcal{A}} \Omega^{1}(\mathcal{A})
$$

We shall say that the metric is symmetric if

$$
g \circ \sigma \propto g .
$$

Of the examples we have discussed we mention that the Connes-Lott model has a unique metric [98]. The matrix models have (more-or-

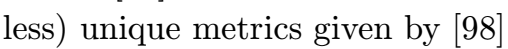

$$
g\left(\theta^{a} \otimes \theta^{b}\right)=g^{a b}, \quad g^{a b} \in \mathbb{C} .
$$

The line $\mathbb{R}_{q}^{1}$ has a (unique) metric which gives rise to an observable: the distance between the spectral lines of $x$ is uniform [1] 5 i]. Other definitions of a metric have been given, some of which are similar to that given above but which weaken

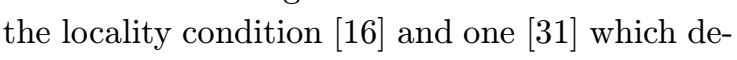
fines a metric on the associated space of states.

\section{Linear Connections}

An important geometric problem is that of comparing vectors and forms defined at two different points of a manifold. The solution to this problem leads to the concepts of a connection and covariant derivative. There are two approaches. The traditional approach considers the connection as a primary object and the covariant derivative is defined in terms of it. But from the point of view of noncommutative geometry, which places primary importance on the algebra of functions, it is the second approach which is the more convenient and is the one which we shall consider here; the covariant derivative is defined as a linear map between modules which satisfies certain Leibniz rules. We shall not define a noncommutative generalization of a connection as a 1-form on a principal fibre bundle [100]

We shall use here the expressions connection and covariant derivative synonymously. In fact we shall distinguish three different types of connections. A left connection or Yang-Mills connection is a connection on a left $\mathcal{A}$-module; it satisfies a left Leibniz rule. A bimodule connection is a connection on a general bimodule $\mathcal{M}$ which satisfies a left and a right Leibniz rule. In 
the particular case where $\mathcal{M}$ is the module of 1 -forms we shall speak of a linear connection.

Let $\mathcal{A}$ be an arbitrary algebra and $\left(\Omega^{*}(\mathcal{A}), d\right)$ a differential calculus over $\mathcal{A}$. One defines $[\overline{7} \overline{9}, \overline{2} \overline{2} \overline{7}]$ a Yang-Mills connection on a left $\mathcal{A}$-module $\mathcal{H}$ as a map

$$
\mathcal{H} \stackrel{D}{\longrightarrow} \Omega^{1}(\mathcal{A}) \otimes_{\mathcal{A}} \mathcal{H}
$$

from a left $\mathcal{A}$-module $\mathcal{H}$ to the tensor product $\Omega^{1}(\mathcal{A}) \otimes_{\mathcal{A}} \mathcal{H}$, which satisfies a left Leibniz rule

$$
D(f \psi)=d f \otimes \psi+f D \psi, \quad f \in \mathcal{A}, \quad \psi \in \mathcal{H} .
$$

It has a natural extension

$$
\Omega^{*}(\mathcal{A}) \otimes_{\mathcal{A}} \mathcal{H} \stackrel{D}{\longrightarrow} \Omega^{*}(\mathcal{A}) \otimes_{\mathcal{A}} \mathcal{H}
$$

given by

$$
D(\alpha \otimes \psi)=d \alpha \otimes \psi+(-1)^{n} \alpha \otimes D \psi
$$

if $\alpha \in \Omega^{n}(\mathcal{A})$.

One defines the field strength by

$$
\Omega \psi=D^{2} \psi
$$

It satisfies the locality condition

$$
\Omega(f \psi)=f \Omega \psi
$$

A linear connection is a covariant derivative

$$
\Omega^{1}(\mathcal{A}) \stackrel{D}{\longrightarrow} \Omega^{1}(\mathcal{A}) \otimes_{\mathcal{A}} \Omega^{1}(\mathcal{A})
$$

on the $\mathcal{A}$-bimodule $\Omega^{1}(\mathcal{A})$ with an extra right Leibniz rule

$$
D(\xi f)=\sigma(\xi \otimes d f)+(D \xi) f
$$

defined using the flip $\sigma$ introduced in (5.1).

We define the torsion map

$$
\Theta: \Omega^{1}(\mathcal{A}) \rightarrow \Omega^{2}(\mathcal{A})
$$

by $\Theta=d-\pi \circ D$. It is left-linear. A short calculation yields

$$
\Theta(\xi) f-\Theta(\xi f)=\pi \circ(1+\sigma)(\xi \otimes d f) .
$$

We shall impose the condition

$$
\pi \circ(\sigma+1)=0
$$

on $\sigma$. It could also be considered as a condition on the product $\pi$. In fact in ordinary geometry it is the definition of $\pi$; a 2-form can be considered as an antisymmetric tensor. Because of this condition the torsion is a bilinear map.

Using $\sigma$ one can also construct an extension

$$
\mathcal{M} \otimes_{\mathcal{A}} \mathcal{M} \stackrel{D_{2}}{\longrightarrow} \Omega^{1}(\mathcal{A}) \otimes_{\mathcal{A}} \mathcal{M} \otimes_{\mathcal{A}} \mathcal{M}
$$

by

$$
D_{2}(\xi \otimes \eta)=D \xi \otimes \eta+\sigma_{12} \circ(\xi \otimes D \eta) .
$$

We have here used the notation

$$
\sigma_{12}=\sigma \otimes 1
$$

which we shall use again below. The operator $D_{2} \circ D$ is not in general left-linear. However, from the condition on $\sigma$ follows the relation

$$
D(\xi \otimes \eta)=\pi_{12} \circ D_{2}(\xi \otimes \eta)+\Theta(\xi) \otimes \eta
$$

between the $D$ given in the extension $(6 . \overline{1})$ and $D_{2}$. It follows that

$$
D^{2}=\pi_{12} \circ D_{2} \circ D+\Theta .
$$

The left-hand side of this equation is defined for a left $\mathcal{A}$-connection whereas the right-hand side is defined only in the case of a linear connection. In particular one can conclude that $\pi_{12} \circ D_{2} \circ D$ is left-linear.

We introduce the notion of metric-compatibility exactly as in the commutative case. Let $g$ be a metric and $(D, \sigma)$ a linear connection. Both $g_{23} \circ D_{2}(\xi \otimes \eta)$ and $d g(\xi \otimes \eta)$ are elements of $\Omega^{1}(\mathcal{A})$. The linear connection is metric-compatible if

$$
g_{23} \circ D_{2}=d \circ g .
$$

We mentioned above the universal calculus over an algebra $\mathcal{A}$. In this case the product $\pi$ is the identity map and it follows that $\sigma=-1$. The ordinary differential $d_{u}$ is clearly a covariant derivative. The torsion vanishes and so does the curvature. Conversely let $D$ define an arbitrary linear connection on the 1 -forms of the universal calculus. If we require the torsion to vanish then $D=d_{u}$. The only torsion-free linear connection is the trivial one. Return to the example of Section 3.1. A covariant derivative is given by

$$
D \xi=-\eta \otimes \xi+\sigma(\xi \otimes \eta), \quad \xi \in \Omega^{1}(\mathcal{A}) .
$$


One can show [98.] that it is unique. The torsion vanishes. The matrix models have a (more-orless) unique torsion-free metric compatible connections. The line $\mathbb{R}_{q}^{1}$ has two connections [1]

Using $\sigma$ a reality condition on the metric and the linear connection can be introduced [5 $\left.5 \overline{1}_{1}^{\prime}\right]$. A reality condition on the curvature can be formulated if $\sigma$ satisfies the braid relation

$$
\sigma_{12} \sigma_{23} \sigma_{12}=\sigma_{23} \sigma_{12} \sigma_{23}
$$

We do not insist upon this for reasons explained in the following section.

\section{Gravity}

The classical gravitational field is normally supposed to be described by a torsion-free, metriccompatible linear connection on a smooth manifold. One might suppose that it is possible to formulate a noncommutative theory of (classical/quantum) gravity by replacing the algebra of functions by a more general algebra and by choosing an appropriate differential calculus. We however mentioned in the first section the problem in defining curvature invariants. One way of circumventing this problem is to consider classical gravity as an effective theory and the EinsteinHilbert action as an induced action. We recall that the classical gravitational action is given by

$$
S[g]=\mu_{P}^{4} \Lambda_{c}+\mu_{P}^{2} \int R .
$$

In the noncommutative case there is a natural definition of the integral $[\overline{3} \overline{2}, \overline{2}, \overline{2}, \overline{2}, \overline{2} \overline{2}]$ but there does not seem to be a natural generalization of the Ricci scalar. One of the problems is the one we touched upon in the preceding section: the natural generalization of the curvature form is in general not right-linear in the noncommutative case. The Ricci scalar then will not be local. One way of circumventing these problems is to return to an old version of classical gravity known as

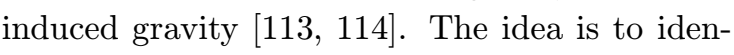
tify the gravitational action with the quantum corrections to a classical field in a curved background. If $\Delta[g]$ is the operator which describes the propagation of a given mode in presence of a metric $g$ then one finds that, with a cut-off $\Lambda$, the effective action is given by

$$
\begin{aligned}
\Gamma[g] & \propto \operatorname{Tr} \log \Delta[g] \simeq \\
& \Lambda^{4} \operatorname{Vol}(V)[g]+\Lambda^{2} S_{1}[g]+(\log \Lambda) S_{2}[g]+\cdots .
\end{aligned}
$$

If one identifies $\Lambda=\mu_{P}$ then one finds that $S_{1}[g]$ is the Einstein-Hilbert action. A problem with this is that it can be only properly defined on a compact manifold with a metric of euclidean signature and Wick rotation on a curved spacetime is a rather delicate if not dubious procedure. Another problem with this theory, as indeed with the gravitational field in general, is that it predicts an extremely large cosmological constant. The expression $\operatorname{Tr} \log \Delta[g]$ has a natural generalization to the noncommutative case [700 See also Example 7.3.5 of Madore [ [91 1 in.

We have defined gravity using a linear connection, which required the full bimodule structure of the $\mathcal{A}$-module of 1 -forms. We argued that this was necessary to obtain a satisfactory definition of locality as well as a reality condition. It is possible to relax these requirements and de-

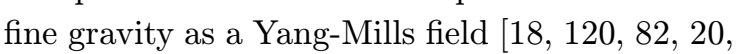
24,5 or as a couple of left and right connections $\left[\overrightarrow{3} \bar{\sigma}_{1}, \overline{3} \overline{7}\right]$. If the algebra is commutative (but not an algebra of smooth functions) then to a cer-

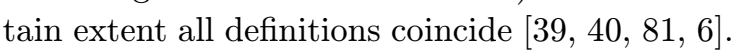

\section{Kaluza-Klein theory}

We mentioned in the Introduction that one of the first, obvious applications of noncommutative geometry is as an alternative hidden structure of Kaluza-Klein theory. This means that one leaves space-time as it is and one modifies only the extra dimensions; one replaces their algebra of functions by a noncommutative algebra, usually of finite dimension to avoid the infinite tower of massive states of traditional Kaluza-Klein theory. Because of this restriction and because the extra dimensions are purely algebraic in nature the length scale associated with them can be arbitrary [92'], indeed as large as the Compton wave length of a typical massive particle.

The algebra of Kaluza-Klein theory is therefore, for example, a product algebra of the form

$$
\mathcal{A}=\mathcal{C}(V) \otimes M_{n}
$$


Normally $V$ would be chosen to be a manifold of dimension four, but since much of the formalism which we shall outline is identical to that of

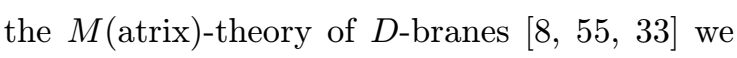
shall leave the dimension unspecified. We mention first electromagnetism and then gravity.

Let $\theta^{i}=\left(\theta^{\alpha}, \theta^{a}\right)$ be a moving frame on $V$. This means that we suppose that $V$ is parallelizable. The matrix factor is also parallelizable. We discussed in some detail in Section 3.2 the case where the differential calculus is based on a set of derivations which form the Lie algebra of $S U_{n}$. According to the discussion of Section 3.7 this can be generalized. One can choose a differential calculus such that

$$
\Omega^{1}\left(M_{n}\right) \simeq \bigoplus_{1}^{d} M_{n}
$$

for arbitrary integer $d$, provided the consistency condition (13.3i) is satisfied. In fact the interesting case is when

$$
n \gg d \text {. }
$$

We write $\Omega^{1}(\mathcal{A})$ as a direct sum

$$
\Omega^{1}(\mathcal{A})=\Omega_{h}^{1} \oplus \Omega_{v}^{1}
$$

with

$$
\begin{aligned}
& \Omega_{h}^{1}=\Omega^{1}(V) \otimes M_{n}, \\
& \Omega_{v}^{1}=\mathcal{C}(V) \otimes \Omega^{1}\left(M_{n}\right) .
\end{aligned}
$$

The differential $d f$ of an element $f$ of $\mathcal{A}$ is given by

$$
d f=d_{h} f+d_{v} f .
$$

In terms of the frame $\theta^{i}$ we have as usual

$$
d_{h} f=e_{\alpha} f \theta^{\alpha}, \quad d_{v} f=e_{a} f \theta^{a}=-[\theta, f] .
$$

We introduce a torsion-free linear connection, compatible with the frame.

$$
d \theta^{i}+\omega^{i}{ }_{j} \theta^{j}=0
$$

with $\theta^{\alpha}+\omega^{\alpha}{ }_{\beta} \theta^{\beta}=0$, and $\omega^{a}{ }_{b}=-\frac{1}{2} C^{a}{ }_{b c} \theta^{c}$. Referring back to Section 4.2 we see that the electromagnetic action for the potential $\omega=A+(\theta+\phi)$ is given by

$$
S[A, \phi]=\int \mathcal{L}(A, \phi)=\frac{1}{4} \operatorname{Tr} \int \Omega_{i j} \Omega^{i j}
$$

$$
\begin{aligned}
& =\frac{1}{4} \operatorname{Tr} \int F_{\alpha \beta} F^{\alpha \beta} \\
& +\frac{1}{2} \operatorname{Tr} \int D_{\alpha} \phi_{a} D^{\alpha} \phi^{a}-\int V(\phi)
\end{aligned}
$$

where [5 $[53$

$$
V(\phi)=-\frac{1}{4} \operatorname{Tr}\left(\Omega_{a b} \Omega^{a b}\right) .
$$

As an example choose $d=3$ and

$$
C_{a b c}=r^{-1} \epsilon_{a b c} .
$$

The hidden 'space' is a fuzzy sphere of radius $r$. The potential $V(\phi)$ vanishes when $\phi$ lies on a gauge orbit of a representation of $S U_{2}$. There are

$$
p(n) \simeq \frac{e^{\pi \sqrt{2 n / 3}}}{4 n \sqrt{3}}
$$

such orbits.

If one replaces the matrix algebra by the algebra of the Connes-Lott model then one obtains a family of theories which includes the standard model of the electroweak interactions. It, and its extensions to include the strong interactions,

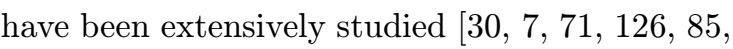
110!.

For the simple models with a matrix extension one can use as gravitational action the Einstein-Hilbert action in 'dimension' $4+d$, including possibly Gauss-Bonnet terms $\left[\overline{8} \overline{8}_{1}^{\prime}, \overline{9} \overline{2}, \overline{9} \overline{1} 1, \overline{9} \overline{3}\right.$, 73 . For a more detailed review we refer to a lecture [94,] at the 5th Hellenic school in Corfu.

\section{String Theory}

Last, but not least, is the possible relation of noncommutative geometry to string theory. We have mentioned that since noncommutative geometry is pointless a field theory on it will be divergencefree. In particular monopole configurations will have finite energy, provided of course that the geometry in which they are constructed can be approximated by a noncommutative geometry, since the point on which they are localized has been replaced by an volume of fuzz, This is one characteristic that it shares with string theory. Certain monopole solutions in string theory have finite energy [1-25] since the point in space (a $D$ brane) on which they are localized has been replaced by a throat to another 'adjacent' $D$-brane. 
In noncommutative geometry the string is replaced by a certain finite number of elementary volumes of 'fuzz', each of which can contain one quantum mode. Because of the nontrivial commutation relations the 'line' $\delta q^{\mu}=q^{\mu \prime}-q^{\mu}$ joining two points $q^{\mu \prime}$ and $q^{\mu}$ is quantized and can be characterized [203in by a certain number of creation operators $a_{j}$ each of which creates a longitudinal displacement. They would correspond to the rigid longitudinal vibrational modes of the string. Since it requires no energy to separate two points the string tension would be zero. This has not much in common with traditional string theory. :- We saw in the previous section that noncommutative Kaluza-Klein theory has much in common with the $M$ (atrix) theory of $D$-branes. What is lacking is a satisfactory supersymmetric extension. Finally we mention that there have been speculations that string theory might give rise naturally to space-time uncertainty relations [84, commutative theory of gravity.

\section{References}

[1] T. Ackermann, J. Tolksdorf, "A generalized Lichnerowicz formula, the Wodzicki Residue and Gravity", IJ. Geom. Phys. 19 (1996) 143.

[2] A. Aghamohammadi, "The two-parametric extension of $h$ deformation of $G L(2)$ and the differential calculus on its quantum plane", 'Mod.'

1. - Phys. Lett. A 8 8 (1993) 2607!

[3] A. Aghamohammadi, M. Khorrami, A. Shariati, " $h$-deformation as a contraction of $q$-deformation", J. Phys. A: Math. Gen. 28, (1995) L225.

[4] P. Aschieri, L. Castellani, "Bicovariant calculus on twisted $I S O(N)$, Quantum Poincaré Group and Quantum Minkowski Space", 'Int. J. Mod.' - - - Phys. A 11 1 (1996) 4513 .

- -[]. J. - A. de _Azcárraga. _P.P. Kulish. _F. Rodenas,

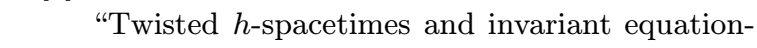
s", 'Z. Physik C $\mathbf{7 6}$ (1997) 567.

[6] A.P. Balachandran, G. Bimonte, G. Landi, F. Lizzi, P. Teotonio-Sobrinho, "Lattice Gauge Fields and Noncommutative _Geometry", 2 - J' ' - _ _ Geom. Phys. 24 (1998) 353.

[7] B.S. Balakrishna, F. Gürsey, K.C. Wali, "Towards a Unified Treatment of Yang-Mills and Higgs Fields", 'Phys. Rev. D 44 (1991) 3313"
[8] T. Banks, W. Fischler, S.H. Shenker, L. Susskind, " $M$ Theory as a Matrix Model: a Conjecture", Phys. Rev. D_55 (1997)_5112.

[9] F. Berezin, "General Concept of Quantization", 'Comm. Math. Phys. 40 (1975) 153'

[10] G. Bimonte, F. Lizzi, G. Sparano, "Distances on a Lattice from Noncommutative Geometry", 'Phys. Lett. B 341 (1994) 139'.

[11] M. Bordemann, J. Hoppe, P. Schaller, M. Schlichenmaier, " $g l(\infty)$ and Geometric Quantization", 'Lomm. Math. Phys. 138 (1991) 209'.

[12] M. Cahen, S. Gutt, J. Rawnsley, "Quantization of Kähler Manifolds I", 'J. Geom. Phys. 7 (1990) $4 \overline{5}$.

[13] U. Carow-Watamura, S. Watamura, "Chirality and Dirac Operator on Noncommutative Sphere", ,Comm. Math. Phys. 183 (1997) 365.

[14] B.L. Cerchiai, J. Wess, " $q$-Deformed Minkowski Space based on a $q$-Lorentz Algebra", Munich Preprint LMU-TPW 98/02, q-alg/9801104:

[15] B.L. Cerchiai, R. Hinterding, J. Madore, J. Wess "The Geometry of a $q$-deformed Phase Space", Euro. J. of Phys. C (to appear).

[16] —, "A Calculus Based on a $q$-deformed Heisenberg Algebra", Euro. J. of Phys. C (to appear).

[17] M. Chaichian, A. Demichev, P. Prešnajder, "Quantum Field Theory on Noncommutative Space-Times and the Persistence of Ultraviolet Divergences", Helsinki Preprint HIP-1998$77 / \mathrm{TH}$, hep-th/9812180

[18] A.H. Chamseddine, G. Felder, J. Fröhlich, "Gravity in Non-Commutative _ Geometry", Comm. Math. Phys. 155 (1993) 205!

[19] A.H. Chamseddine, A. Connes, "Universal Formula for Noncommutative Geometry Actions: Unification of Gravity and the Standard Model", "Phys. Rev. Lett. $\mathbf{7} \overline{7}(\overline{1} 996)-\overline{8} 68^{\prime}$.

[20] A.H. Chamseddine, J. Fröhlich, O. Grandjean, "The gravitational sector in the Connes-Lott formulation of the standard model", ' Phys._36 (1995) 625

[21] G. Chapline, N.S. Manton, "The Geometrical Significance of Certain Higgs Potentials: An approach to grand unification", 'Nucl. Phys. B 184 (1980) 391.

[22] S. Cho, J. Madore, K.S. Park, "Noncommutative Geometry of the $h$-deformed Quantum Plane", J. Phys. A: Math. Gen. 31 (1998) 2639. 
[23] S. Cho, R. Hinterding, J. Madore, H. Steinacker, "Finite Field Theory on Noncommutative GeL - - ometries", Munich Preprint,LMU-TPW 99-06, hep-th/9903239.

[24] Chong-Sun Chu, Pei-Ming Ho, B. Zumino, "Some Complex Quantum Manifolds and their

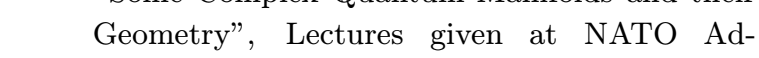
vanced Stùdy Institute on Quantum Fields and Quantum Space Time, Cargese, France, 1996, hep=th/9608-188

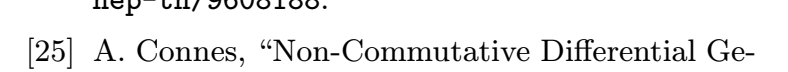
ometry", Publications of the Inst. des Hautes

, - - - Etudes Scientifiques. 62 _(1986) 257.

'- [26\} A. -Gonnes,- "Fhe Actien -Funetiertad -in - Nert- Commutative Geometry", "Comm. Math. Phys.' - $\mathbf{1 1 7}(\mathbf{1 9 8 8 )} 673$.

[27] A. Connes, "Noncommutative Geometry", Academic Press, 1994.

[28] A. Connes, "Noncommutative geometry and reality", iJ. Math. Phys. $\mathbf{3 6}(1995) 6194$

- [29]- A. Connes, -Gravity- coupled-with matter -and

- - - the fotludation-of-non-cemmutative-geemetry", Comm. Math. Phys. 192 (1996) 155

[30] A. Connes, J. Lott, "Particle Models and Noncommutative Geometry", in 'Recent Advances in Field Theory', 'Nucl. Phys. 18 (Proc. Suppl.) : -

[31] —, "The metric aspect of non-commutative geometry", Proceedings of the 1991 Cargèse Summer School, Plenum Press, 1992.

[32] A. Connes, M.A. Rieffel, "Yang-Mills for noncommutative two-tori", Contemp. Math. 62 (1987) 237.

[33] A. Connes, M.R. Douglas, A. Schwarz, "Noncommutative Geometry and Matrix Theory: Compactification on Tori", 'J. High Energy

: - - - - - - -

; f34ł -R.- Goquereaux; - "Noncommutative- geonretry ' - - and -theoretiead - phrysics", - If.-Geom: -Phys.- 6 - .

[35] R. Coquereaux, R. Häußling, F. Scheck, "Algebraic connections on parallel universes", 'Int. J.' : - Mod. Phys. A A 10 (1995) $8 \overline{9}$

[36] A. Cuntz, D. Quillen, "Algebra extensions and nonsingularity", J. Amer. Math. Soc. 8 (1995) 251.

[37] L. Dabrowski, P.M. Hajac, G. Landi, P. Siniscalco, "Metrics and pairs of left and right connections on bimodules", 'J. Math. Phys. 37.' (1996) 4635
[38] D. de Wit, J. Hoppe, H. Nicolai, "On the Quantum Mechanics of Supermembranes", 'Nucl.' Phys. B 305 (1988) 545!

[39] A. Dimakis, F. Müller-Hoissen, "A Noncommutative Differential Calculus and its Relation to Gauge theory and Gravitation", "Int. $\bar{J}$. Mod.' Phys. A 3 (1993) 474.

[40] —, "Discrete Differential Calculus, Graphs, Topologies and Gauge Theory", 'J. Math. Phys.' $\mathbf{3 5}-\overline{1994)}-\overline{6} \overline{03}$.

[41] A. Dimakis, J. Madore, "Differential Calculi and Linear Connections", "J. Math. Phys. $\mathbf{3} \overline{7}(\overline{1996)}$ $46 \overline{4} 7$.

[42] P.A.M. Dirac, "The Fundamental Equations of Quantum Mechanics", Proc. Roy. Soc. A109 (1926) 642; "On Quantum Algebras", Proc. Camb. Phil. Soc. 23 (1926) 412.

[43] S. Doplicher, K. Fredenhagen, J.E. Roberts, "The Quantum Structure of Spacetime at the Planck Scale and Quantum Fields", "Comm.' Math. Phys. 172 $(1995)$ 187.

[44] M. Dubois-Violette, R. Kerner, J. Madore, "Gauge bosons in a noncommutative geometry", Phys. Lett. B_217 (1989) 485.

[45] M. Dubois-Violette, J. Madore, T. Masson, J. Mourad, "Linear Connections on the Quantum Plane", 'Lett. Math. Phys. 35_(1995) 351'.

[46] M. Dubois-Violette, J. Madore, T. Masson, J. Mourad, "On Curvature in Noncommutative Geometry", "J. Math. Phys._37_(1996)_4089'.

[47] L.D. Faddeev, N.Y. Reshetikhin, L.A. Takhtajan, "Quantization of Lie Groups and Lie Algebras", Alge. i Analy. 1 (1989) 178; Leningrad Math. J. 1 (1990) 193.

[48] M. Fichtmüller, A. Lorek, J. Wess, " $q$-Deformed Phase Space and its Lattice Structure", ' $\bar{Z}$. .' Physik C $\mathbf{7 1}(1996)$ 533.

[49] T. Filk, "Divergences in a field theory on quantum space", iPhys. Lett. B $\mathbf{3} \overline{\mathbf{7}} \mathbf{6}(1996) 53$.

[50] G. Fiore, " $S O_{q}(N)$-isotropic Harmonic Oscillator on the Quantum Euclidean Space $\mathbb{R}_{q}^{N-1}$,, Proceedings of the Clausthal Symposium on Nonlinear, Dissipative, Irreversible Quantum Systems, 1994.

[51] G. Fiore, J. Madore, "Leibniz Rules and Reality Conditions, Preprint 98-13, Dip. Matematica e Applicazioni, Università di Napoli, q-alg/9806071; 
[52] —, "The Geometry of the Quantum Euclidean Space", Munich Preprint LMU-TPW 97-23, g-alg/9904027!

[53] P. Forgács, N.S. Manton, "Space-Time Symmetries in Gauge Theories", 'Comm. Math. Phys.' $-\overline{72}(19 \overline{8} 0)-15$

[54] J. Fröhlich, O. Grandjean, A. Recknagel, "Supersymmetric quantum theory, noncommutative geometry and gravitation", Lecture Notes, Les Houches 1995, hep-th/9706132!.

[55] O.J. Ganor, S. Ramgoolam, W. Taylor, "Branes, Fluxes and Duality in $M$ (atrix)Theory, 'Nucl. Phys. B 492 (1997) 191!

[56] L.J. Garay, "Quantum Gravity and Minimum Length", Int. J. Mod. Phys. A 10 (1995) 145!

[57] B. Greene, S.-T. Yau (Eds.), "Mirror Symmetry II", AMS/IP Studies in Advanced Mathematics, 1997.

[58] H. Grosse, J. Madore, "A Noncommutative Version of the Schwinger Model", 'Phys. Lett. B $\mathbf{2} \mathbf{8 3}$ ",

: - $(1992)-218$

[59] H. Grosse, P. Prešnajder, "The Construction of Noncommutative Manifolds Using Coherent States", 'Lett. Math. Phys. $\mathbf{2 8}(\overline{1993)} 239$ '.

' $[60]==$ "The Dirac Operator on the Fuzzy Sphere" Lett. Math. Phys. 33 (1995) 171'

[61] H. Grosse, G. Reiter, "The Fuzzy Supersphere", math-ph/9804013.

[62] H. Grosse, C. Klimčík, P. Prešnajder, "Towards Finite Quantum Field Theory in noncommutative Geometry", Int. J. Theor. Phys. 35 (1996) 231.

[63] —, "Topological Nontrivial Field Configurations in Noncommutative Geometry", 'Comm. Math.'

:- - Phys. $\mathbf{1} \overline{7} \mathbf{8}(1997)-507$.

[64] —, "On 4D Field Theory in Non-Commutative Geometry", 'Comm. Math. Phys. 180 (1997)', $---\overline{4} 29$

[65] —, "Field Theory on a Supersymmetric Lattice", 'Comm. Math. Phys. $185(1997) 155$.

[66] E.J. Hellund, K. Tanaka, "Quantized SpaceTime", 'Phys. Rev. 94 (1954) 192.'.

[67] Pei-Ming Ho, Yong-Shi Wu, "Noncommutative Geometry and D-branes", iPhys. Lett. B 398!

- - - (1997) 52

[68] J. Hoppe, "Diffeomorphism groups, Quantization and $S U(\infty)$ ", Int. J. Mod. Phys. A

(1989) 5235
[69] A. Jevicki, S. Ramgoolam, "Non commutative gravity from the ADS/CFT correspondence", Brown-Het-1159, hep-th/9902059,

[70] W. Kalau, M. Walze, "Gravity, NonCommutative Geometry and the Wodzicki Residue", IJ. Geom. Phys. 16 (1995) 327.

[71] D. Kastler "A detailed account of Alain Connes' version of the standard model in noncommutative geometry", Rev. Math. Phys. 5 (1993) 477.

[72] D. Kastler, J. Madore, D. Testard, "Connections of bimodules in noncommutative geometry, Contemp. Math. 203 (1997) 159.

[73] A. Kehagias, J. Madore, J. Mourad, G. Zoupanos, "Linear Connections in Extended Space-Time", 'J. Math. Phys. $\mathbf{3 6}$ (1995) 5855.

[74] A. Kehagias, P. Meessen, G. Zoupanos, "Deformed Poincaré Algebra and Field Theory" Phys. Lett. B 346 (1995) 262.

[75] A. Kempf, G. Mangano, "Minimal Length Uncertainty Relation and Ultraviolet Regularization", 'Phys. Rev. D_55 (1997)_7909.

[76] A. Kempf, G. Mangano, R.B. Mann, "Hilbert Space Representation of the Minimal Length Uncertainty Relation", 'Phys. Rev. D_52 (1995)', 1108.

[77] M. Khorrami, A. Shariati, A. Aghamohammadi, "SL $L_{h}(2)$-Symmetric Torsionless Connections", Lett. Math. Phys. 40 (1997) 95.

[78] T. Krajewski, R. Wulkenhaar, "Perturbative Quantum Gauge Fields on the Noncommutative Torus", Marseille preprint CPT-99/P.3794, hep-th/9903187.

[79] J.L. Koszul, "Lectures on Fibre Bundles and Differential Geometry", Tata Institute of Fundamental Research, Bombay, 1960.

[80] B.A. Kupershmit, "The quantum group $G L_{h}(2)$ ", J. Phys. A 25 (1992) L1239.

[81] G. Landi, "An Introduction to Noncommutative Spaces and their Geometries", Springer Lecture Notes, Springer-Verlag, 1997.

[82] G. Landi, Ai Viet Nguyen, K.C. Wali, "Gravity and electromagnetism in noncommutative geometry", 'Phys. Lett. B $\mathbf{3 2 6}(1994)-\overline{5}$.

[83] F. Leitenberger, "Quantum Lobachevsky Planes", IJ. Math. Phys. 37 1996$)$ 3131.'.

[84] M. Li, T. Yoneya, "Short Distance Space-Time Structure and Black Holes in String Theory: a Short Review of the Present Status", EFI-98-24, hep-th/9806240 
[85] F. Lizzi, G. Mangano, G. Miele, G. Sparano, "Constraints on Unified Gauge Theories from Noncommutative Geometry", 'Mod. Phys. Lett.' : - _ - 11 (1996) 2561!

[86] J. Madore, "Non-Commutative Geometry and the Spinning Particle", Proceedings of the XI Warsaw Symposium on Elementary Particle Physics 'New Theories in Physics', May 1988,

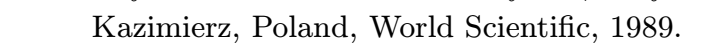

[87] —, "Kaluza-Klein Aspects of Noncommutative Geometry", Proceedings of the XVII Inter-

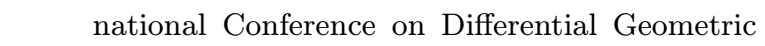

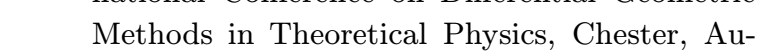
gust, 1988. World Scientific, 1989.

[88] _ , "Modification of Kaluza-Klein Theory", Phys. Rev. D 41 (1990)_3709.

[89] —, "The Commutative Limit of a Matrix Geometry", "J. Math. Phys. 32 (1991) 332, "The Fuzzy

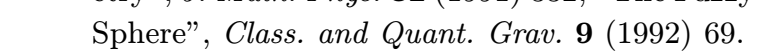

- - - - - - - 201 -

[ $[90]$ _. "Fuzzy Physics", IAnn. Phys. (NY) 219' - _ _ (1992) 187?

[91] —, "An Introduction to Noncommutative Differential Geometry and its Physical Application-

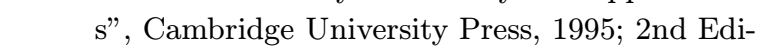

- - - tiōn, 1999.'-

[92] J Madore, J. Mourad, "Algebraic-Kaluza-Klein Cosmology", 'Class. and Quant. Grav. 10 (1993)'

: - - - - - 2157 ;

[93] —, "On the Origin of Kaluza-Klein Structure",

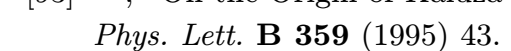

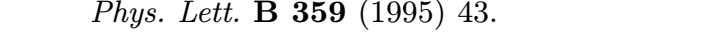

[94] -, "Noncommutative Kaluza-Klein Theory", Lecture given at the 5th Hellenic School and

'- Workshops on Elementary Particle Physics, hep-th $/ 9601169$

[95] —, "Quantum Space-Time and Classical Gravity", 'J. Math. Phys. 39.1998) 423'

[96] J. Madore, J. Mourad, A. Sitarz, "Deformations of Differential Calculi" 'Mod. Phys. Lett. A 12' (1997) 975 .

[97] J. Madore, L.A. Saeger, "Topology at the Planck Length", 'Class. and Quant. Grav. 15! - -

[98] J. Madore, T. Masson, J. Mourad, "Linear Connections on Matrix Geometries", 'Class. and" Quant. Grav. 12 (1995) 1429

[99] S. Majid, "Hopf Algebras for physics at the Planck scale", 'Class. and Quant. Grav. 5 (1988)' i- - - - 1587
[100] —, "Quantum Geometry and the Planck Scale", DAMTP Preprint, q-alg/9701001".

[101] Yu. I. Manin, "Quantum Groups and Noncommutative Geometry", Les Publications du Centre de Recherche Mathématiques, Montréal, 1988.

[102] —, "Multiparametric Quantum Deformations of the General Linear Supergroup", 'Comm.' Math. Phys. 123 (1989) 163

[103] N.S. Manton, "A New Six-Dimensional Approach to the Weinberg-Salam Model", 'N $\bar{N} u \bar{c}$.' Phys. B 158 (1979) 141'

[104] C.P. Martín, D. Sánchez-Ruiz, "The Oneloop UV-Divergent Structure of $U(1)$-YangMills Theory on Noncommutative $\mathbb{R}^{4}$ ", , Madrid Preprint FT/UCM-15-99, hep-th/9903077'

[105] J. Mourad, "Linear Connections in NonCommutative Geometry", 'Class. and Quant.' Grav. 12 (1995) 965.

[106] C. Ohn, "A *-Product on $S L(2)$ and the Corresponding Nonstandard Quantum- $U(\mathfrak{s l}(2))$ ", LEtt. Math. Phys. 25_(1992) 85.

[107] P. Podleś, "Quantum Spheres", 'Lett. 'Math.' Phys. 47 (1987) 193.

[108] P. Podleś, "Solutions of the Klein-Gordon and Dirac Equation on Quantum Minkowski Spaces", '-Comm. Math. Phys. 182 (1996) 569!

[109] P. Podleś, S.L. Woronowicz, "On the Classification of Quantum Poincaré Groups", 'Comm.' Math. Phys. 178 (1996) $6 \overline{1}_{\text {. }}$

[110] I. Pris, T. Schücker, "Noncommutative geometry beyond the standard model", 'J. Math. Phys." $38(1997) 2255$.

[111] M.A. Rieffel, "Non-commutative Tori - A case study of Non-commutative Differentiable Manifolds", Contemp. Math. 105 (1980) 191.

[112] —, " $C^{*}$-algebras associated with irrational rotation", Pacific J. Math. 93 (1981) 415.

[113] A.D. Sakharov, "Vacuum quantum fluctuations in curved space and the theory of gravitation", Doklady Akad. Nauk S.S.S.R. 177 (1967) 70.

[114] A.D. Sakharov, "Spectral Density of Eigenvalues of the Wave Equation and Vacuum Polarization", Teor. i Mat. Fiz. 23 (1975) 178.

[115] K. Schmüdgen, "Operator representations of a $q$-deformed Heisenberg algebra", Leibzig Preprint, 'q-alg/9805131'. 
[116] P. Schupp, P. Watts, B. Zumino, "The 2-Dimensional Quantum Euclidean Algebra", LEett. Math. Phys. 24 (1992) 141'.

[117] J. Schwenk, J. Wess, "A q-deformed quantum mechanical toy model", 'Phys. Lett. B 291' i - (1992) 273

[118] A. Hebecker, S. Schreckenberg, J. Schwenk, W. Weich, J. Wess, Representations of a q-deformed Heisenberg algebra, 'Z. Physik $\mathbf{C} \mathbf{6 4}(19 \overline{9} \overline{4}) 35 \overline{5}$.

[119] J. Schwinger, "Unitary Operator Bases", Proc. Nat. Acad. Sci. 46, (1960) 570.

[120] A. Sitarz, "Gravity from non-commutative geometry", 'Class. and Quant. Grav. 11 (1994)' ; - - - 2127

[121] J.J. Sylvester, "Lectures on the Principles of Universal Algebra", Amer. J. Math. 6 (1884) 271.

[122] H.S. Snyder, "Quantized Space-Time", Pי-Phys.' : - Rev. 71 (1947) 38;

[123] —, "The Electromagnetic Field in Quantized Space-Time", 'Phys. Rev. $\mathbf{7 2}(1947) 68$

[124] G. 't Hooft, "Quantization of point particles in (2+1)-dimensional gravity and spacetime discreteness", 'Class. and Quant. Grav. 13.1996)'

;

[125] J.P. Gauntlett, J. Gomis, P.K. Townsend, "BPS Bounds for Worldvolume Branes", Energy Phys. 9801 (1998) 003"

[126] J.C. Várilly, J.M. Gracia-Bondía, "Connes' noncommutative differential geometry and the Standard Model", 'J. Geom. Phys. 12 (1993)! $---\overline{2} 3$

[127] J. Wess, B. Zumino, "Covariant Differential Calculus on the Quantum Hyperplane", 'Nucl.' ' - - Phys. 18 (Proc. Suppl.) (1990) 302'

[128] S.L. Woronowicz, "Twisted $S U(2)$ Group. An example of a Non-Commutative Differential Calculus", Publ. RIMS, Kyoto Univ. 23 (1987) 117; "Compact Matrix Pseudogroups", 'Comm.' '- Math. Phys. 111 (1987) 613.

[129] H. Weyl, "The Theory of Groups and Quantum Mechanics", Dover, New York, 1950, 